\title{
Teager-Kaiser energy detector for narrowband wireless microphone spectrum sensing
}

\author{
Matthieu Gautier, Marc Laugeois and Dominique Noguet \\ CEA, LETI, Minatec, Grenoble, France \\ matthieu.gautier1@cea.fr
}

\begin{abstract}
In this paper, the wireless microphone sensing is addressed for a TV white space communication and we aim to achieve an efficient semi-blind detection of narrowband FM modulation signals. To this end, the proposed solution is to use the Teager-Kaiser energy operator which takes into account the non-linear model of speech. Then, a filterbank based technique splits the analysis of the wideband signal into subbands in order to increase the accuracy of the algorithms.

Simulation results show that a $4 \mathrm{~dB}$ detection gain could be achieved by the Teager-Kaiser energy detector compared to the energy detector. The subbands decomposition leads to a further $4 \mathrm{~dB}$ improvement. Experimental measurements allow a realistic validation of the proposed solutions.
\end{abstract}

\section{INTRODUCTION}

The performance of sensing algorithms is fundamental to establish the opportunistic communication of a cognitive radio (CR) system [1]. The UHF band is a candidate under-used band where the CR system could operate [2]. In this band, the primary users are the TV transmitters and the wireless microphones. If the detection of TV signals has been addressed in literature, solutions need to be proposed for the detection of the wireless microphones.

Unlike the detection of digital TV signals which can use the characteristics of the OFDM modulation [3], the wireless microphone sensing is difficult due to the few characteristics of its signal. Most of the literature references use a blind detection for the specific case of the wireless microphones [4]. These studies are based on an eigenvalue decomposition [5], a spectral correlation [6] and an energy detector [7].

One of the common properties of these algorithms is that they assume the detection of wideband signals. However, the European TV band is composed of 48 channels of $8 \mathrm{MHz}$ bandwidth. Each band has to be analysed for the detection of both the TV signals and the microphones and a decision variable will be provided to the CR system which takes the final decision of using one band or not. The analysed TV channel is wide compared to the frequency band occupied by the microphone signal. Thus, an important assumption of our study is that the detection of the wireless microphones deals with narrowband signals.

In this paper, a semi-blindly detector is proposed, it uses the Frequency Modulation (FM) characteristic of the wireless microphone signal. This method is based on the Teager-Kaiser energy operator [8] which takes into account the non-linear model of a FM signal energy. This operator allows an accurate estimation of the energy. Then, a second approach is tested, the analysis of the wideband signal is splitted into subbands in order to increase the accuracy of the algorithms.

This paper consists of 5 parts. Following this introduction, Section II gives the models of the wireless microphone signals and of the CR system. This section also includes a state of the art of the blind detectors of the wireless microphones. In Section III, the Teager-Kaiser energy operator is introduced and the narrowband solutions are presented. Simulation results are given in this part. Section IV details an experimental validation of the proposed solutions. Finally, conclusions are drawn and outlook is provided.

\section{WIRELESS MICROPHONE SENSING}

\section{A. The wireless microphones model}

The wireless microphones operate in the UHF band. Most of them use an analog FM modulation [4]. The signal has a spectral bandwidth $B_{x}$ of $200 \mathrm{kHz}$. But, most of the signal energy is concentrated in a bandwidth of $40 \mathrm{kHz}$. The transmit power is a few tens of $\mathrm{mW}$. The coverage area is therefore relatively low, about 500 meters for the most powerful microphones. Their detection is difficult to achieve. The signal from the microphone $x(t)$ can be modeled as follows:

$$
x(t)=A \cos \left(2 \pi f_{0} t+\frac{\kappa_{f}}{s_{m}} \int_{\tau} s(\tau) d \tau\right),
$$

where $f_{0}$ is the carrier frequency, $\kappa_{f}$ the frequency deviation of the FM modulation, and $s(t)$ the modulating signal having an amplitude $s_{m}$. The signal $x(t)$ has a power $\sigma_{x}^{2}$ equals to $A^{2} / 2$.

Let $y(t)$ be the microphone signal received by the opportunist receiver and $n(t)$ an Additive White Gaussian Noise (AWGN) with a zero mean and a variance $\sigma_{n}^{2}$ :

$$
y(t)=x(t)+n(t)
$$

$x(t)$ and $n(t)$ being independant, the Signal to Noise Ratio $(S N R)$ received by the opportunistic user is:

$$
S N R=\frac{\sigma_{x}^{2}}{\sigma_{n}^{2}}=\frac{A^{2}}{2 \sigma_{n}^{2}} .
$$

\section{B. The cognitive radio system description}

The function that the CR detector has to perform is the one of detecting signals in the presence of noise, which can be 

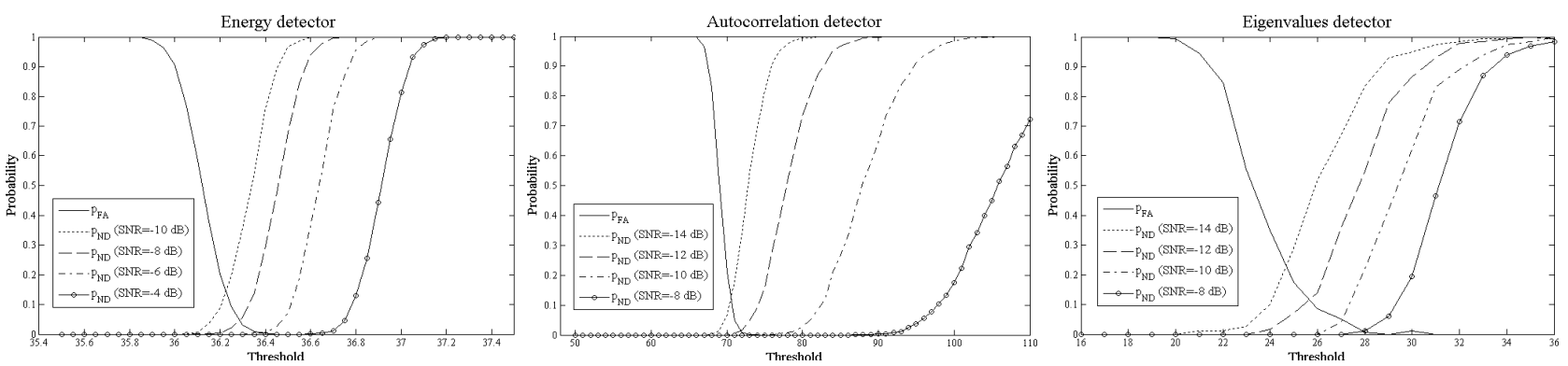

Fig. 1. False alarm and non-detection probabilities of the classical detectors.

stated as the following hypothesis:

$$
\left\{\begin{array}{l}
H_{0}: y(t)=n(t) \\
H_{1}: y(t)=x(t)+n(t)
\end{array}\right.
$$

where $H_{0}$ is the null hypothesis for the event "free band" and $H_{1}$ is the alternative hypothesis for the event "occupied band". By choosing one of the two assumptions $H_{0}$ and $H_{1}$, two kinds of errors can occur:

- the false alarm error: it corresponds to the case when the hypothesis $H_{1}$ is chosen while the band is free. The false alarm probability is denoted by $p_{F A}$.

- the non-detection error: it occurs when the hypothesis $H_{0}$ is chosen while the band is occupied. The non-detection probability is denoted by $p_{N D}$.

The performance of the detectors is evaluated by comparing the probabilities $p_{F A}$ and $p_{N D}$ depending on the absolute threshold of detection. The $p_{N D}$ are given for different powers of the received signal in order to determine the minimum $S N R$ that can be detected.

In this study, the detection band is a TV UHF channel, its bandwidth $B_{c}$ is equal to $8 \mathrm{MHz}$ in Europe and $6 \mathrm{MHz}$ in US. We consider that there is at most one wireless microphone in the observation band.

The baseband microphone signal is generated following the model of Eq. (1) with these parameters: the frequency bandwidth of $s(t)$ is $20 \mathrm{kHz}$, the FM deviation $\kappa_{f}$ is 3 , the carrier frequency $f_{0}$ is equal to $100 \mathrm{kHz}$ and the noise power $\sigma_{n}^{2}$ is set to $0 \mathrm{dBm}$. Under these conditions, the transmitted microphone signal $x(t)$ has a frequency bandwidth around $100 \mathrm{kHz}$.

Based on the European UHF band specifications, the observation window is $8 \mathrm{MHz}$ (narrowband conditions) and the algorithm considers $N=4096$ samples (approximately $0.5 \mathrm{~ms}$ for $8 \mathrm{MHz}$ ).

\section{State of the art of the blind detection}

A selection of three classical blind detectors is presented. Two of them have been studied in the case of microphone sensing [5][7]. These methods lie on a stationary and deterministic model of the signal mixed with a stationary noise.

1) Energy detection: The energy detector computes a variable which is proportional to the energy of the received signal [9][10]. The test statistic $T$ of the energy detector is given by:

$$
T=\frac{1}{N} \sum_{n=0}^{N-1}|y(n)|^{2},
$$

where $N$ the number of samples of the analyzed signal.

The test statistic is then compared to a threshold. The way the absolute thresh is set is out of the scope of this paper.

2) Autocorrelation based detection: This method tests the stationarity of the signal calculates the samples autocorrelation function. In the noise-only case, the autocorrelation function should approach a Dirac impulse since the noise is white. The receiver estimates the autocorrelation function $r(k)$ of the received signal:

$$
r(k)=E\left[y(n) y^{*}(n-k)\right], \quad k=0, \pm 1, \pm 2, \ldots \pm N,
$$

where $E[]$ is the mathematical expectation operator and * represents the complex conjugation.

There are several test statistics that can be used. The most efficient one [11] uses the sum of the square of the amplitude of all components of the autocorrelation function and the square of the amplitude of the central sample $(k=0)$. Thus, the following decision variable is calculated:

$$
T=\frac{\sum_{k=0}^{N-1}|r(k)|^{2}}{|r(0)|^{2}} .
$$

When the signal is white, the 2 terms should be roughly equal, since the non-central values $(k \neq 0)$ should be approximately zero. When the wireless microphone signal is present, the signal is white and the statistical test should increase.

3) Eigenvalues based detection: Another blind sensing technique uses the eigenvalues of the correlation matrix [5]. First, the detector estimates the autocorrelation function of the received signal. Then, the correlation matrix is given by:

$$
R=\left(\begin{array}{ccc}
r(0) & \ldots & r(N-1) \\
r(1) & \ldots & r(N-2) \\
\vdots & \ddots & \vdots \\
r(N-1) & \ldots & r(0)
\end{array}\right)
$$

Let $\lambda_{i}$ be the eigenvalues of matrix $R$.

Several test statistics have been tested [11]. Both of these test statistics are a measure of the non-whiteness of the spectrum. 


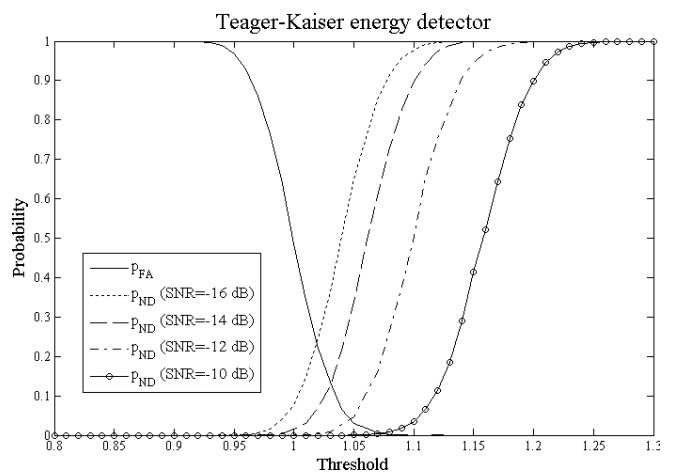

Fig. 2. False alarm and non-detection probabilities of the Teager-Kaiser energy detector.

In the following, we use the ratio of the largest eigenvalue and the smallest eigenvalue:

$$
T=\lambda_{\max } / \lambda_{\min } .
$$

4) Simulated performance: Fig. 1 shows the performance of the three previous algorithms. The false alarm probability $p_{F A}$ and non-detection probability $p_{N D}$ are computed versus the detection threshold for different $S N R$ of the primary transmitted signal (among $S N R$ between $-14 \mathrm{~dB}$ and $-4 \mathrm{~dB}$ ). In narrowband conditions and for an analysis duration of 0.5 ms $(N=4096)$, the simulation results show that:

- the energy detector enables the detection of $S N R$ down to $-6 \mathrm{~dB}$,

- the autocorrelation based detector enables the detection of $S N R$ down to $-12 \mathrm{~dB}$,

- the eigenvalues based detector enables the detection of $S N R$ down to $-8 \mathrm{~dB}$.

In the following, the eigenvalues based detector will not be considered due to its implementation complexity compared to the others while its performance is not better.

\section{SENSING ALGORITHMS FOR NARROWBAND WIRELESS MICROPHONE SIGNALS}

In this section, two approaches are tested to overcome the problem of wireless microphone sensing. The most innovative solution is to take into account the FM modulation characteristic of the signal and to use the appropriate TeagerKaiser energy operator. Then, we propose to decompose the narrowband detection into several wideband detections by using a filterbank.

\section{A. Teager-Kaiser energy detector}

We propose a new method for energy detection suitable to detect microphones. Instead of using the conventional energy detector, we propose to use the Teager-Kaiser energy operator to measure the energy activity of a sample. This operator better reflects the energy of FM signal.

Introduced in 90 [8], Kaiser uses the results of Teager and Teager (especially the energy curve needed to produce speech) which showed the non-linear model of the speech. These changes of the characteristics of the speech signal can be

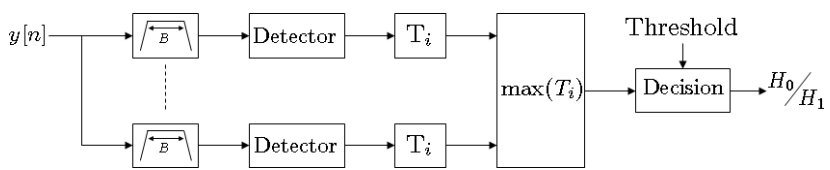

Fig. 3. Structure of the narrowband filterbank based detector.

modeled as a linear combination of AM-FM signals.

Based on this model, Kaiser has proposed an very simple and fast algorithm [8] to estimate energy, called the Teager-Kaiser energy operator, since the restriction related to the bandwidth of the signal (narrowband signal) is respected.

One of the first applications of this operator is the detection of FM modulations.

The Teager-Kaiser energy operator $\Psi$ extracts directly the energy from the instantaneous signal and is expressed by:

$$
\Psi[x(k)]=[x(k)]^{2}-x(k+1) x(k-1) .
$$

Adding a white Gaussian noise $n(t)$, the operator becomes:

$$
\begin{aligned}
\Psi[y(k)] & =\Psi[x(k)+n(k)], \\
& =\Psi[x(k)]+\Psi[n(k)]+2 \Psi[x(k), n(k)] .
\end{aligned}
$$

with $\Psi[x(k), n(k)]$ the cross energy operator defined as:

$$
\begin{aligned}
\Psi[x(k), n(k)]=x(k) \cdot n(k) & -\frac{1}{2} x(k+1) n(k-1) \\
& -\frac{1}{2} x(k-1) n(k+1) .
\end{aligned}
$$

$\Psi[x(k), n(k)]=0$ if $x(t)$ and $n(t)$ are uncorrelated. Then, the average value of the operator of $y(k)$ is:

$$
\begin{aligned}
E\langle\Psi[y(k)]\rangle & =E\langle\Psi[x(k)]\rangle+E\langle\Psi[n(k)]\rangle, \\
& =E\langle\Psi[x(k)]\rangle+\sigma_{n}^{2} .
\end{aligned}
$$

In Section II, we have seen that the wireless microphone is a FM modulation of a speech signal, so the Teager-Kaiser energy detector should be adapted to this kind of signal. From Eq. (15), the semi-blind detection could be performed by computing the test statistic $T=E\langle\Psi[y(k)]\rangle$.

Fig. 2 shows the performance of the Teager-Kaiser energy detector. The false alarm probability $p_{F A}$ and non-detection probability $p_{N D}$ are computed versus the threshold for different $S N R$ of the primary transmitted signal $(S N R=-16 \mathrm{~dB}$, $-14 \mathrm{~dB},-12 \mathrm{~dB}$ and $-10 \mathrm{~dB}$ ).

The simulation results show that, the Teager-Kaiser energy detector can detect SNR down to $-10 \mathrm{~dB}$. This algorithm should be compared with the energy detection algorithm in order to check if the energy model is non-linear and if this detector provides a better estimate of a FM signal energy. So, compared to the performance of Fig. 1, the Teager-Kaiser energy detector allows a $4 \mathrm{~dB}$ gain of detection.

Note that this detector does not induce an important increase of the complexity compared to the energy detector.

\section{B. Adapted counterparts of the wideband algorithms}

The narrowband detection is decomposed into wideband detections by filtering the signal into subbands. Fig. 3 describes the structure of the adapted detector using a filterbank 

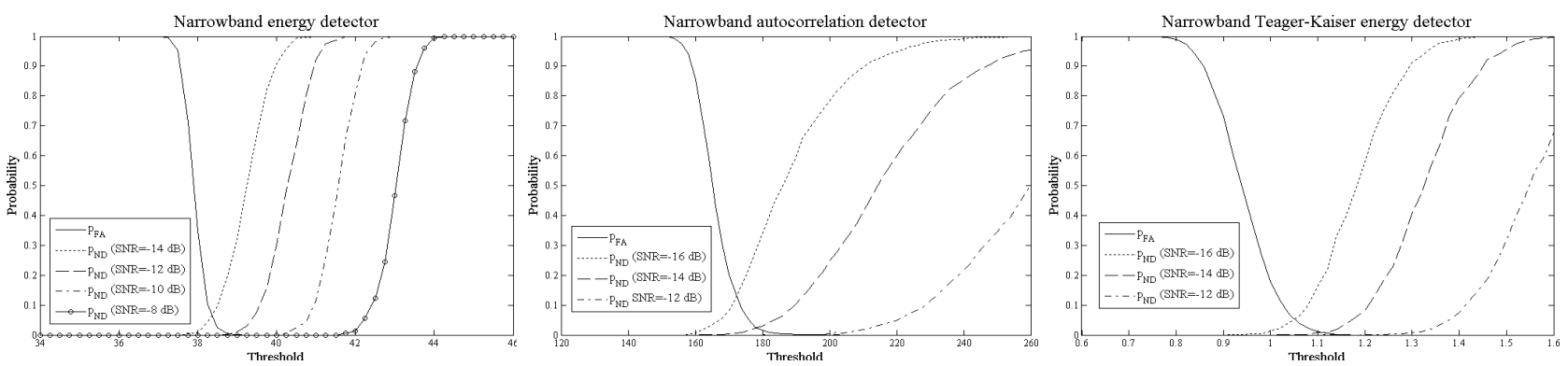

Fig. 5. False alarm and non-detection probabilities of the narrowband algorithms.
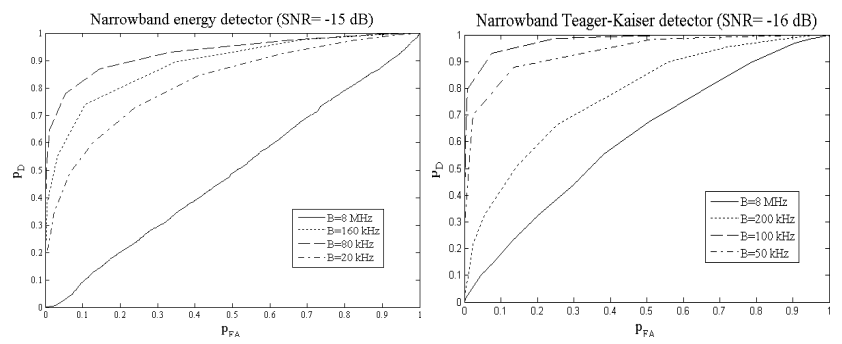

Fig. 4. Detection probability versus false alarm probability for different window bandwidths of the narrowband power detection and the narrowband Teager-Kaiser detection.

structure. Once filtered, each subband is processed by a detector and a test statistic $T_{i}$ is computed for each subband. The final decision is made on the maximum of these test statistics. This structure depends on the filters' bandwidth $B$. As detectors, we propose to use the energy detector, the autocorrelation detector and the Teager-Kaiser energy detector.

In the specific case of the energy detector [12], the energy calculation could be performed in the frequency domain. The filterbank is replaced by a Fast Fourier Transform (FFT) which analyzes the spectral content of the $N$-samples signal. The FFT size is chosen to have sufficient frequency resolution in the signal band:

$$
\frac{B_{c}}{N_{F F T}}<<B_{x},
$$

where $N_{F F T}$ is the size of the transform and $B_{x}$ the microphone signal bandwidth in the channel bandwidth $B_{c}$. Once the FFT is performed, the energy detection is computed on each subband. This algorithm is called the "narrowband energy detector".

For the autocorrelation detector and the Teager-Kaiser energy detector, the test statistic must be computed in the time domain, so these detectors must use a filterbank as shown in Fig. 3. These new algorithms are called the "narrowband autocorrelation detector" and the "narrowband Teager-Kaiser energy detector".

For the three structures, the optimum width $B$ of the frequency window has to be set. Obtained by simulation, the probability of detection $p_{D}$ are plotted versus the false alarm probability
$p_{F A}$ for different frequency bandwidths. Performance is compared to the one obtained with the algorithm without filtering (curves $B=8 \mathrm{MHz}$ ). The results are shown in Fig. 4:

- For the narrowband energy detection using a FFT, the curves are obtained for $S N R=-15 \mathrm{~dB}$ and for $B$ set from $20 \mathrm{kHz}$ to $160 \mathrm{kHz}$. First, these simulations show the improvement of the new algorithm. Indeed, when the windowing is not used, detection is clearly impossible. Then, the results show that the size of the sliding window gives the best results for $80 \mathrm{kHz}$. When the bandwidth is too small or too large compared to the signal bandwidth, performance is degraded. In the following, a bandwidth of $80 \mathrm{kHz}$ will be used.

- For the autocorrelation algorithm using a filterbank, simulation results are not displayed in this paper. However, the optimum bandwidth has been computed and a bandwidth of $400 \mathrm{kHz}$ is used in the following.

- For the narrowband Teager-Kaiser energy detector, the curves are obtained for $S N R=-16 \mathrm{~dB}$ and for $B=50 \mathrm{kHz}$, $100 \mathrm{kHz}$ and $200 \mathrm{kHz}$. These simulations show that the detection is clearly impossible when the filterbank is not used. Then, the results show that a bandwidth of $100 \mathrm{kHz}$ provides the best results, this value will be used thereafter.

\section{Simulation results}

Fig. 5 shows the performance of the three narrowband algorithms. The false alarm probability $p_{F A}$ and non-detection probability $p_{N D}$ are computed versus the detection threshold for different $S N R$ of the primary transmitted signal (between $-16 \mathrm{~dB}$ and $-8 \mathrm{~dB})$.

Compared to the simulation results of Fig. 1, the simulation results show that:

- the narrowband energy detector enables the detection of signals with $S N R=-12 \mathrm{~dB}$, leading to a $6 \mathrm{~dB}$ improvement of the energy detector,

- the narrowband autocorrelation detector enables the detection of signals with $S N R=-14 \mathrm{~dB}$, leading to a $2 \mathrm{~dB}$ improvement of the autocorrelation detector,

- the narrowband Teager-Kaiser energy detector enables the detection of signals with $S N R=-14 \mathrm{~dB}$, leading to a 4 $\mathrm{dB}$ improvement of the Teager-Kaiser energy detector.

These algorithms allow an interesting increase of the minimal SNR that could be detected. The FFT based implementation of the narrowband energy detector is a promising solution. 


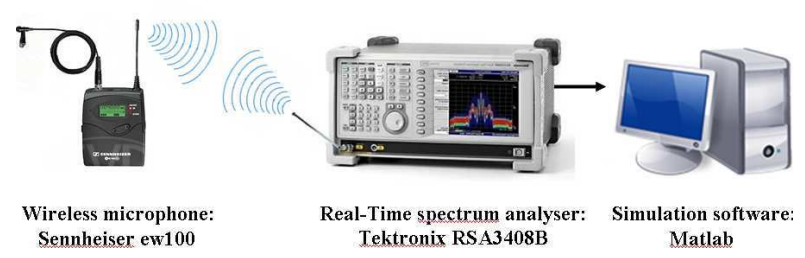

Fig. 6. Experimental test bench of wireless microphone sensing.

\section{EXPERIMENTAL VALIDATION}

In order to test the proposed algorithms on real signals, an experimental validation is presented in this section.

\section{A. System description}

The experimental test bench is described in Fig. 6. It consists of a wireless microphone (Sennheiser ew100) equipped with an UHF antenna and a microphone, it can transmit on 1440 frequencies (with a step of $25 \mathrm{kHz}$ ) between 518 and 866 $\mathrm{MHz}$ at a power of $15 \mathrm{dBm}$. The signal from the microphone is received by the spectrum analyzer Tektronix RSA 3408B. This analyzer can record baseband signals on a $12.8 \mathrm{MHz}$ band (in our case). The line-of-sight channel is considered to be an AWGN one. Using the Matlab software, the recorded signals are then processed by the algorithms described previously.

\section{B. Experimental results}

Measurements are recorded for different powers of the microphone signal. For each record, the detection probability is computed (leading to a false alarm probability of $10 \%$ ) for various detectors. Results are shown in Fig. 7, the detection probability $p_{D}$ is plotted versus the different received SNR.

The measurement results are consistent with those obtained by simulation. The curves with squares show that the narrowband algorithms outperform their initial counterparts. The measures also show the improvement of the use of the TeagerKaiser energy operator instead of the energy detector.

\section{CONCLUSIONS}

In this paper, the detection of narrowband wireless microphone signals has been addressed. The motivation of this study is to indentify a wireless microphone detector that could be implemented with a low complexity. This paper focuses on the performance of the different algorithms.

The first point revealed in this study is the performance of the promising Teager-Kaiser energy detector. Taking into account the non-linear model of the FM signal energy, this operator leads to a $4 \mathrm{~dB}$ gain compared to the classical energy detector without an important increase of the complexity. The narrowband detectors based on subbands decomposition allow a 2 to $6 \mathrm{~dB}$ improvement of the classical detectors. In the case of the narrowband energy detector, its implementation could be based on the FFT operator inducing a low complexity.

Future work concerns the computation of the complexity of the different algorithms in order to choose the detectors with the best performance-complexity tradeoff. The relative threshold calculation should be also studied for a material implementation.

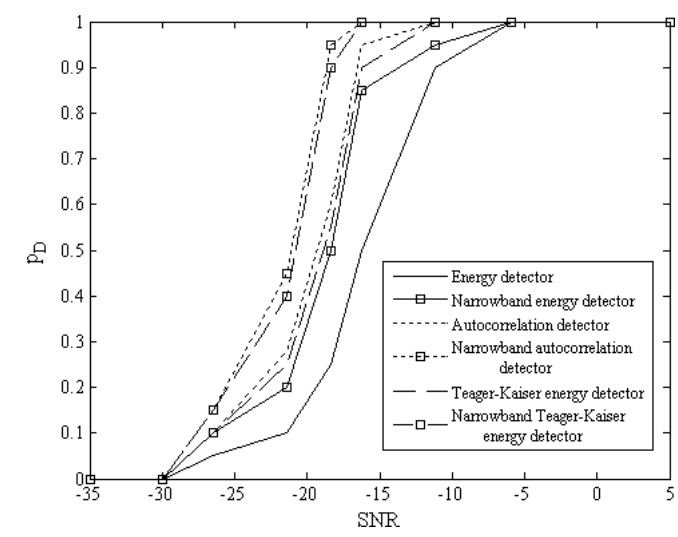

Fig. 7. Measured detection probability versus SNR for various detectors.

\section{ACKNOWLEDGMENT}

The research leading to these results was derived from the EU 7th Framework Programme (FP7) ICT-QoSMOS and the French national project ANR-INFOP.

\section{REFERENCES}

[1] J. Mitola III and G. Q. Maguire Jr, "Cognitive radio: making software radios more personal," IEEE Personal Communications, vol. 6, no. 4, pp. 13-18, 1999.

[2] Official announcement of FCC, "FCC adopts rules for unlicensed use of television white spaces," available from http://www. ffC.gov, November 2008.

[3] A. Bouzegzi, P. Jallon, and P. Ciblat, "A second order statistics based algorithm for blind recognition of OFDM based systems," IEEE Global Communications Conference (GLOBECOM2008), December 2008.

[4] H.-S. Chen, W. Gao, and D. Daut, "Spectrum sensing for wireless microphone signals," IEEE Sensor, Mesh and Ad Hoc Communications and Networks Workshops (SECON08), June 2008.

[5] S. Xu, Y. Shang, and H. Wang, "SVD based Sensing of a Wireless Microphone Signal in Cognitive Radio Networks," IEEE International Conference on Communications Systems, November 2008.

[6] N. Han, S. H. Shon, J. O. Joo, and J. M. Kim, "Spectral correlation based signal detection method for spectrum sensing in IEEE 802.22 WRAN systems," International Conference on Advanced Communication Technology, February 2006.

[7] M. Ghosh, V. Gaddam, G. Turkenich, and K. Challapali, "Spectrum Sensing Prototype for Sensing ATSC and Wireless Microphone Signals," International Conference on Cognitive Radio Oriented Wireless Networks and Communications (CROWNCOM08), May 2008.

[8] J. F. Kaiser, "On simple algorithm to calculate the energy of signal," IEEE International Conference on Acoustics, Speech, and Signal Processing (ICASSP90), April 1990.

[9] H. Urkowitz, "Energy detection of unknown deterministic signals," Proceeding of the IEEE, vol. 55, no. 4, pp. 523-531, April 1967.

[10] A. Sonnenschein and P. M. Fishman, "Radiometric detection of spreadspectrum signals in noise of uncertain power," IEEE Transactions on Aerospace and Electronic Systems, vol. 28, no. 3, July 1992.

[11] S. J. Shellhammer, "Spectrum sensing in IEEE 802.22," IAPR Workshop on Cognitive Information Processing, June 2008.

[12] N. M. Neihart, S. Roy, and D. J. Allstot, "A parallel multi-resolution sensing technique for multiple antenna cognitive radios," IEEE International Symposium on Circuits and Systems, May 2007. 